\title{
How To Write Mathematical Equations, Expressions, and Symbols with LaTeX: A cheatsheet.
}

\author{
Authorea Help
}

April 28, 2020

\section{What is LaTeX?}

LaTeX is a programming language that can be used for writing and typesetting documents. It is especially useful to write mathematical notation such as equations and formulae.

\section{How to use LaTeX to write mathematical notation}

There are three ways to enter "math mode" and present a mathematical expression in LaTeX:

1. inline (in the middle of a text line)

2. as an equation, on a separate dedicated line

3. as a full-sized inline expression (displaystyle)

\section{$2.1 \quad$ inline}

Inline expressions occur in the middle of a sentence. To produce an inline expression, place the math expression between dollar signs ( $\$$ ). For example, typing $\$ \mathrm{E}=\mathrm{mc} \wedge 2 \$$ yields $E=m c^{2}$.

\section{2 equation}

Equations are mathematical expressions that are given their own line and are centered on the page. These are usually used for important equations that deserve to be showcased on their own line or for large equations that cannot fit inline. To produce an inline expression, place the mathematical expression between the symbols $\backslash[$ and $\backslash]$. Typing $\backslash\left[x=\backslash f \operatorname{rac}\left\{-b \backslash p m \backslash \operatorname{sqrt}\left\{b^{\wedge} 2-4 a c\right\}\right\}\{2 a\} \backslash\right]$ yields

$$
x=\frac{-b \pm \sqrt{b^{2}-4 a c}}{2 a}
$$

\section{3 displaystyle}

To get full-sized inline mathematical expressions use \displaystyle. Typing I want this $\$ \backslash$ displaystyle $\backslash \operatorname{sum}_{-}\{n=1\}^{\wedge}\{\backslash$ $\backslash \operatorname{frac}\{1\}\{\mathrm{n}\} \$$, not this $\$ \backslash$ sum $_{-}\{\mathrm{n}=1\}^{\wedge}\{\backslash \operatorname{infty}\} \backslash \operatorname{frac}\{1\}\{\mathrm{n}\} \$$. yields: I want this $\sum_{n=1}^{\infty} \frac{1}{n}$, not this $\sum_{n=1}^{\infty} \frac{1}{n}$. 


\section{Symbols (in math mode)}

\subsection{The basics}

As discussed above math mode in LaTeX happens inside the dollar signs (\$. .\$), inside the square brackets $\backslash[\ldots \backslash]$ and inside equation and displaystyle environments. Here's a cheatsheet showing what is possible in a math environment:

\begin{tabular}{|c|c|c|}
\hline description & command & output \\
\hline addition & + & + \\
\hline subtraction & - & - \\
\hline plus or minus & $\backslash \mathrm{pm}$ & \pm \\
\hline multiplication (times) & Itimes & $x$ \\
\hline multiplication (dot) & Icdot & . \\
\hline division symbol & $\backslash$ div & $\div$ \\
\hline division (slash) & / & / \\
\hline simple text & $\backslash$ text $\{$ text $\}$ & text \\
\hline infinity & \infty & $\infty$ \\
\hline dots & $1,2,3, \backslash 1$ dots & $1,2,3, \ldots$ \\
\hline dots & $1+2+3+\backslash \operatorname{cdots}$ & $1+2+3+$. \\
\hline fraction & $\backslash \operatorname{frac}\{a\}\{b\}$ & $\frac{a}{b}$ \\
\hline square root & $\backslash \operatorname{sqrt}\{x\}$ & $\sqrt{x}$ \\
\hline$n$th root & $\backslash$ sqrt $[\mathrm{n}]\{\mathrm{x}\}$ & $\sqrt[n]{x}$ \\
\hline exponentiation & $a^{\wedge} b$ & $a^{b}$ \\
\hline subscript & $a_{-} b$ & $a_{b}$ \\
\hline absolute value & $|x|$ & $|x|$ \\
\hline natural log & $\backslash \ln (x)$ & $\ln (x)$ \\
\hline logarithms & $\backslash \log _{-}\{a\} b$ & $\log _{a} b$ \\
\hline exponential function & $e^{-} x=\backslash \exp (x)$ & $e^{x}=\exp (x)$ \\
\hline $\operatorname{deg}$ & $\backslash \operatorname{deg}(f)$ & $\operatorname{deg}(f)$ \\
\hline degree & \degree & \\
\hline $\operatorname{arcmin}$ & - \prime & ' \\
\hline $\operatorname{arcsec}$ & $-\{\backslash$ prime \prime $\}$ & " \\
\hline circle plus & \oplus & $\oplus$ \\
\hline circle times & lotimes & $\otimes$ \\
\hline equal & $=$ & $=$ \\
\hline not equal & Ine & $\neq$ \\
\hline less than & $<$ & $<$ \\
\hline less than or equal to & $\backslash l e$ & $\leq$ \\
\hline greater than or equal to & lge & $\geq$ \\
\hline approximately equal to & lapprox & $\approx$ \\
\hline
\end{tabular}

\section{$3.2 \quad$ Functions}

$\begin{array}{lll}\text { description } & \text { command } & \text { output } \\ \text { maps to } & \text { \to } & \rightarrow \\ \text { composition } & \text { \circ } & \circ\end{array}$




\subsection{Greek and Hebrew letters}

\begin{tabular}{|c|c|c|c|}
\hline command & output & command & output \\
\hline$\backslash$ alpha & $\alpha$ & $\backslash$ tau & $\tau$ \\
\hline Ibeta & $\beta$ & $\backslash$ theta & $\theta$ \\
\hline$\backslash$ chi & $\chi$ & Iupsilon & $v$ \\
\hline$\backslash$ delta & $\delta$ & $\backslash x i$ & $\xi$ \\
\hline \epsilon & $\epsilon$ & $\backslash$ zeta & $\zeta$ \\
\hline \varepsilon & $\varepsilon$ & $\backslash$ Delta & $\Delta$ \\
\hline leta & $\eta$ & $\backslash$ Gamma & $\Gamma$ \\
\hline Igamma & $\gamma$ & $\backslash$ Lambda & $\Lambda$ \\
\hline \iota & $\iota$ & \Omega & $\Omega$ \\
\hline$\backslash$ kappa & $\kappa$ & $\backslash \mathrm{Phi}$ & $\Phi$ \\
\hline$\backslash$ lambda & $\lambda$ & $\backslash \mathrm{Pi}$ & $\Pi$ \\
\hline$\backslash \mathrm{mu}$ & $\mu$ & $\backslash \mathrm{Psi}$ & $\Psi$ \\
\hline$\backslash n u$ & $\nu$ & $\backslash$ Sigma & $\Sigma$ \\
\hline \omega & $\omega$ & $\backslash$ Theta & $\Theta$ \\
\hline$\backslash p h i$ & $\phi$ & IUpsilon & $\Upsilon$ \\
\hline \varphi & $\varphi$ & $\backslash X X i$ & $\Xi$ \\
\hline$\backslash p i$ & $\pi$ & $\backslash$ aleph & $\aleph$ \\
\hline$\backslash p s i$ & $\psi$ & Ibeth & $\beth$ \\
\hline \rho & $\rho$ & \daleth & 7 \\
\hline \sigma & $\sigma$ & \gimel & I \\
\hline
\end{tabular}

\subsection{Vectors}

$\begin{array}{lll}\text { description } & \text { command } & \text { output } \\ \text { vector } & \backslash \operatorname{vec}\{\mathrm{v}\} & \vec{v} \\ \text { vector } & \backslash \operatorname{mathbf}\{\mathrm{v}\} & \mathbf{v} \\ \text { norm } & || \backslash \operatorname{vec}\{\mathrm{v}\}|| & \|\vec{v}\|\end{array}$




\subsection{Set theory}

\begin{tabular}{|c|c|c|}
\hline description & command & output \\
\hline set brackets & $\backslash\{1,2,3 \backslash\}$ & $\{1,2,3\}$ \\
\hline element of & $\backslash$ in & $\in$ \\
\hline subset of & Isubset & $\subset$ \\
\hline subset of & Isubseteq & $\subseteq$ \\
\hline contains & $\backslash$ supset & $\supset$ \\
\hline contains & Isupseteq & $\supseteq$ \\
\hline union & Icup & $\cup$ \\
\hline intersection & Icap & $\cap$ \\
\hline big union & $\backslash$ bigcup_\{n=1 $\}^{\wedge}\{10\} A_{-} n$ & $\bigcup A_{i}$ \\
\hline big intersection & $\backslash$ bigcap_ $\{n=1\} \leadsto\{10\} A_{-} n$ & $\bigcap A_{n}$ \\
\hline empty set & lemptyset & $\emptyset=$ \\
\hline power set & $\backslash$ mathcal $\{\mathrm{P}\}$ & $\mathcal{P}$ \\
\hline minimum & $\backslash \min$ & $\min$ \\
\hline maximum & $\backslash \max$ & $\max$ \\
\hline supremum & Isup & sup \\
\hline infimum & \inf & $\inf$ \\
\hline limit superior & $\backslash$ limsup & $\limsup$ \\
\hline limit inferior & \liminf & $\liminf$ \\
\hline closure & loverline $\{A\}$ & $\bar{A}$ \\
\hline
\end{tabular}

\subsection{Logic}

$\begin{array}{lll}\text { description } & \text { command } & \text { output } \\ \text { not } & \backslash \text { sim } & \sim \\ \text { and } & \backslash \text { land } & \wedge \\ \text { or } & \backslash \text { lor } & \vee \\ \text { if...then } & \text { \to } & \rightarrow \\ \text { if and only if } & \backslash \text { leftrightarrow } & \leftrightarrow \\ \text { logical equivalence } & \text { \equiv } & \equiv \\ \text { therefore } & \text { \therefore } & \therefore \\ \text { there exists } & \text { \exists } & \exists \\ \text { for all } & \text { \forall } & \forall \\ \text { implies } & \text { \Rightarrow } & \Rightarrow \\ \text { equivalent } & \text { \Leftrightarrow } & \Leftrightarrow\end{array}$




\subsection{Calculus}

\begin{tabular}{|c|c|}
\hline description & command \\
\hline derivative & $\backslash f r a c\{d f\}\{d x\}$ \\
\hline derivative & $\backslash f$ \\
\hline partial derivative & $\backslash f r a c\{\backslash$ partial $f\}\{\backslash$ partial $x\}$ \\
\hline limits & $\backslash \lim _{-}\{x \backslash$ to $\backslash$ infty $\}$ \\
\hline summation & $\backslash \operatorname{sum}_{-}\{n=1\}^{\wedge}\{\backslash$ infty $\} a \_n$ \\
\hline product & $\backslash$ prod_$\{n=1\}^{\wedge}\{\backslash$ infty $\}$ a_n \\
\hline integral & \int \\
\hline double integral & \iint \\
\hline triple integral & \iiint \\
\hline
\end{tabular}

\subsection{Number theory}

$\begin{array}{lll}\text { description } & \text { command } & \text { output } \\ \text { divides } & \mid & \mid \\ \text { does not divide } & \backslash \text { not I } & \chi \\ \text { greatest common divisor } & \text { Igcd } & \text { gcd } \\ \text { ceiling } & \backslash \text { lceil x Irceil } & \lceil x\rceil \\ \text { floor } & \backslash \text { lfloor x \rfloor } & \lfloor x\rfloor\end{array}$

\subsection{Geometry and trigonometry}

\begin{tabular}{|c|c|c|}
\hline description & command & output \\
\hline angle & \angle ABC & $\angle A B C$ \\
\hline degree & $90^{-}\{\backslash \operatorname{circ}\}$ & $90^{\circ}$ \\
\hline triangle & Itriangle $A B C$ & $\triangle A B C$ \\
\hline segment & loverline $\{A B\}$ & $\overline{A B}$ \\
\hline sine & $\backslash \sin$ & $\sin$ \\
\hline cosine & $\mid \cos$ & $\cos$ \\
\hline tangent & $\backslash \tan$ & $\tan$ \\
\hline cotangent & $1 \cot$ & $\cot$ \\
\hline secant & $\backslash \mathrm{sec}$ & $\mathrm{sec}$ \\
\hline cosecant & $\backslash \csc$ & $\csc$ \\
\hline inverse sine & Varcsin & $\arcsin$ \\
\hline inverse cosine & larccos & $\arccos$ \\
\hline iverse tangent & larctan & $\arctan$ \\
\hline
\end{tabular}

\section{Symbols (in text mode)}

The followign symbols do not have to be surrounded by dollar signs. 


$\begin{array}{lll}\text { description } & \text { command } & \text { output } \\ \text { dollar sign } & \backslash \$ & \$ \\ \text { percent } & \backslash \% & \% \\ \text { ampersand } & \backslash \& & \& \\ \text { pound } & \backslash \# & \# \\ \text { backslash } & \backslash \text { textbackslash } & \backslash \\ \text { left quote marks } & \text { ' } & " \\ \text { right quote marks } & ,, & " \\ \text { single left quote } & \text { " } & , \\ \text { single right quote } & , & \text { X-ray } \\ \text { hyphen } & \mathrm{X}-\mathrm{ray} & \text { pp. 5-15 } \\ \text { en-dash } & \text { pp. 5--15 } & \text { Yes-or no? } \\ \text { em-dash } & \text { Yes---or no? } & \end{array}$

\section{$5 \quad$ Writing LaTeX notation in Authorea}

Authorea supports LaTeX writing, but you don't have to know LaTeX to use Authorea! In order to insert LaTeX in an Authorea document:

1. Create a new document

2. Click on the Insert button in the toolbar and then select LaTeX from the dropdown.

Here are some tips for writing LaTeX in Authorea:

1. Click anywhere outside of the LaTeX block to render it.

2. Hover on Preview to see a Preview of the rendered content.

3. Do not paste an entire LaTeX article! Instead import documents from your homepage.

4. Only type LaTeX content in a LaTeX block, i.e. everything you would write after \begin\{document\}. }

5. Do not type preamble (e.g. documentclass), frontmatter, macros or figures.

6. To add macros (newcommands) and packages, click Settings $\rightarrow$ Edit Macros

7. Use the Insert Figure button to insert images (and data).

8. Use math mode for equations, e.g. $\mathcal{L}_{E M}=-\frac{1}{4} F^{\mu \nu} F_{\mu \nu}$.

9. Try the citation tool (click cite) to find and add citations, or use \cite\{\}.

10. To insert more LaTeX blocks click Insert $\rightarrow$ LaTeX.

11. You can use sectioning commands like \section \{\}$, \backslash$ subsection \{\}$, \backslash$ subsubsection \{\} to add headings. ${ }^{1}$

\section{$6 \quad$ Further reading}

Interested in finding out more about LaTeX? Check out these examples of how LaTeX can be used to write mathematical equations, and follow these instructions to create powerful documents in LaTeX on Authorea, beyond mathematics.

\footnotetext{
${ }^{1}$ You can toggle heading numbering on/off from the article settings. This footnote is generated via ${ }^{2}$ |
} 